\title{
Rheology evolution of a geopolymer precursor aqueous suspension during ageing
}

\author{
Gilles Dusserre*, Anaïs Farrugia, and Thierry Cutard \\ Institut Clément Ader (ICA) ; Université de Toulouse ; CNRS, IMT Mines Albi, INSA, ISAE-SUPAERO, \\ UPS ; Campus Jarlard, F-81013 Albi, France
}

\begin{abstract}
Geopolymer based glass-ceramic matrix composites can be processed at room temperature and a heat treatment below $100^{\circ} \mathrm{C}$ leads to matrix hardening thanks to the geopolymerization mechanisms. The stabilization of the matrix into glass-ceramics is achieved via a post-curing at high temperature. This paves the way of the utilization of cost effective liquid composite molding processes, for which all the necessary equipment is already available for processing temperature ranges related to polymer matrix composites, provided that the rheological behavior of the precursor is suitable to conveniently permeate the fibrous preform. The paper describes the thixotropic rheological behavior of a reference suspension at processing temperature $\left(10-20^{\circ} \mathrm{C}\right)$ and its evolution along ageing at $18^{\circ} \mathrm{C}$. The changes are interpreted in terms of geopolymerization mechanisms (dissolution and polycondensation) and suspension rheology (predominance of hydrodynamic effects at high shear rate). On this basis, a phenomenological modeling framework, combining two Krieger-Dougherty equations, is proposed to build a relationship between the effective viscosity of the suspension and
\end{abstract}

\footnotetext{
${ }^{*}$ Corresponding author Gilles Dusserre (Gilles.Dusserre@mines-albi.fr)

IMT Mines Albi - Institut Clément Ader

Campus Jarlard - route de Teillet

81013 ALBI CT cedex 9

Tel. +33 (0) 563493309

Fax. +33 (0) 563493242
} 
the phenomena involved during ageing (dissolution of alumino-silicate particles) and shearing (microstructure scalar variable).

Keywords: geopolymers ; suspensions ; rheology/rheometry ; composite ; thixotropy

\section{Introduction}

Geopolymer based glass-ceramic matrix composites can be processed at room temperature and a heat treatment below $100^{\circ} \mathrm{C}$ leads to matrix hardening thanks to the geopolymerization mechanisms. The stabilization of the matrix into glass-ceramics is achieved via a post-curing at high temperature. The hardening at low temperature makes it possible to use cost effective liquid composite molding processes, without necessitating the development of specific equipment. However it requires that the rheological behavior of the precursor is suitable to conveniently permeate the fibrous preform. Geopolymer precursors are aqueous suspensions of alumino-silicate particles in an alkaline silicate solution ${ }^{1}$. The hardening roughly acts in three main steps ${ }^{2}$, (i) dissolution of the alumino-silicate particles in the alkaline solution, (ii) polycondensation of the dissolved alumino-silicates with the silicates in solution, and (iii) development of a gel phase that grows up to the hardening of the material.

The main issue that hinders the processing of geopolymer precursors by liquid composite molding is the high viscosity of the concentrated suspensions, necessary to achieve a dense enough matrix to match the composite requirements.

Indeed, geopolymer precursors are described as yield fluids ${ }^{3,4}$ whose viscosity is influenced by chemical composition, temperature and history ${ }^{4}$, which means that their flow may require high stresses, that would lead, for instance, to preform deformation or unfilled areas.

Understanding the rheology of concentrated suspensions is still an active field of research, both through simulation ${ }^{5,6}$ and experiment ${ }^{7}$. It is governed by several phenomena (Brownian motion, hydrodynamic effects, colloidal interactions, friction, lubrication), which are more or less predominant depending on the solid fraction and shear rate (or Péclet number) ${ }^{8}$. It results in a nonNewtonian behavior, influenced by the shape, size, chemistry and surface charge of the particles. The particles are likely to adopt a specific arrangement under given conditions ${ }^{7,9}$, and this so-called microstructure influences the effective viscosity of the suspension. If the microstructure setting-up is not instantaneous (i.e. faster than the characteristic time for macroscopic flow), the effective viscosity at a given shear rate progressively changes together with the microstructure build-up, resulting in a thixotropic behavior ${ }^{10}$.

One efficient way to take into account thixotropy is the definition of a scalar microstructure variable, governed by a law of evolution, and which can be deduced from rheology ${ }^{11}$. The law of evolution describes the competition of a microstructure build-up mechanism that tends to increase the 
variable, and a breakdown mechanism that tends to decrease the variable. At least this latter mechanism, depending on the mechanical loading, is defined as a function of shear rate ${ }^{10,12}$, shear stress $^{11,13}$ or dissipated energy ${ }^{14}$.

This paper reports an investigation of the changes in rheological behavior of a geopolymer precursor formulated for pre-impregnated composite plies, in view of the formulation of a suspension suitable for liquid composite molding. The effects on rheology of solid fraction and ageing at storage temperature are interpreted according to the phenomena at work in suspensions. Based upon this interpretation, a phenomenological model provides a global framework able to describe all the characteristics of the rheology of the suspension, and paves the way toward a rheology-based metrics for microstructure description of such a suspension.

\section{Experimental Procedure}

The suspension under study is a non-commercial geopolymer precursor optimized for manufacturing commercial parts or assemblies made of pre-impregnated glass-ceramic matrix composite. Its composition is confidential, but it matches with the previous general description of geopolymer precursors, with particle size lower than $1 \mu \mathrm{m}$ and $\mathrm{Si} / \mathrm{Al}$ ratio much higher than unity. The formulation includes a significant $\mathrm{K}$ content, and to a lesser extent $\mathrm{Na}$ and $\mathrm{Ca}$.

Before processing or characterization, the suspension is stored at $-18^{\circ} \mathrm{C}$ to prevent any hardening. However, the first step of geopolymerization, i.e. dissolution of the alumino-silicate particles in the alkaline solution, is likely to occur during storage, leading to an ageing of the precursor. The reference suspension, from which all the other suspensions are derived, has undergone a short ageing time $\mathrm{T}_{0}$ and is referred to as non-aged.

The effect of solid fraction on the rheology of non-aged precursor is investigated via an addition of 2 wt.\%, 3 wt.\% and 5 wt.\% (respectively 5, 7.5 and 12.5 vol.\%) of distilled water, just before characterization.

Shear rheology characterizations were performed using a rheometer (Rheostress 600, Thermo Electron) in cone-plate test configuration ( $1^{\circ}$ truncated cone with a gap of $0.054 \mathrm{~mm}$ ). Both cone and plate have a smooth surface and a diameter of $35 \mathrm{~mm}$.

Prior to each experiment, the samples are pre-sheared at a shear rate of $70 \mathrm{~s}^{-1}$ for 3 minutes and then kept at rest for 3 minutes in order to provide a reproducible initial state. The rheological characterization consists in steady shear tests, carried out by applying an upward and downward strain rate scan between $6 \cdot 10^{-3} \mathrm{~s}^{-1}$ and $200 \mathrm{~s}^{-1}$, with a dwell-time of 2 minutes at $200 \mathrm{~s}^{-1}$, this whole characterization cycle taking about 15 minutes. The steady stress (change in stress lower than $5 \%$ during $5 \mathrm{~s}$ ) is recorded as a function of strain rate and plotted in Figure 1-4. 
Non-aged samples of suspension were characterized at 10,15 and $20^{\circ} \mathrm{C}$. The characterization cycle described above was performed 3 times after $10 \mathrm{~min}$ at rest between each cycle, in order to follow the rheological evolution in the processing temperature range. About 25 minutes have past between the start of two consecutive cycles. Aged samples of undiluted suspension were characterized at $15^{\circ} \mathrm{C}$ after storage periods at $-18^{\circ} \mathrm{C}$ increasing from $\mathrm{T}_{1}$ to $\mathrm{T}_{5}$. Non-aged samples of suspension diluted with 2,3 and $5 \mathrm{wt} . \%$ of distilled water were characterized at $15^{\circ} \mathrm{C}$.

More details about the experiments are available in reference ${ }^{15}$.

\section{Results}

\section{Rheological behavior of the suspension}

The non-Newtonian behavior of the suspension is first presented. In this section, only rheograms obtained at increasing shear rate are analyzed in order to highlight the changes in rheological behavior. The rheograms obtained at decreasing shear rate are plotted in dashed lines but not analyzed in detail in the present section. The thixotropic behavior is further analyzed in the Discussion section.

In the strain rate range of interest $\left(6 \cdot 10^{-3}\right.$ to $\left.200 \mathrm{~s}^{-1}\right)$, the shear-thinning behavior overall obeys the Carreau law, with a difference of 2 or 3 decades between the effective viscosity on the Newtonian plateau, $\eta_{0}$, and the effective viscosity on the high shear rate plateau, $\eta_{\infty}$, as shown in Figure 1 . In the temperature processing range, between 10 and $20^{\circ} \mathrm{C}$, the viscosity decreases with temperature, from 530 to 212 Pa.s at $6 \cdot 10^{-3} \mathrm{~s}^{-1}$ and from 0.88 to 0.62 Pa.s at $200 \mathrm{~s}^{-1}$. The ratio of viscosities at $200 \mathrm{~s}^{-1}$ between 10 and $20^{\circ} \mathrm{C}$ is 1.4 , close to the value for water, 1.3. This shows that at high shear rate, the viscosity of the suspension is roughly proportional to the viscosity of the fluid phase, i.e. the hydrodynamic interactions predominate. On the other hand, the ratio of viscosities at $6 \cdot 10^{-3} \mathrm{~s}^{-1}$ between 10 and $20^{\circ} \mathrm{C}$ is of 2.5 . This shows that temperature sensitive interactions are at work at low shear rate, which promotes the assumption of significant contribution of Brownian motion.

\section{Rheological behavior evolution at processing temperature}

Figure 2 plots the rheograms measured at 10,15 and $20^{\circ} \mathrm{C}$ for non-aged and non-diluted suspensions and shows how the rheological behavior changes with time in the processing temperature range. At $10^{\circ} \mathrm{C}$ (Figure 2.a), the changes in rheological behavior are barely significant. The viscosity at $6 \cdot 10^{-3}$ $\mathrm{s}^{-1}$ decreases from 530 to 445 Pa.s, whereas the viscosity at $200 \mathrm{~s}^{-1}$ remains between 0.85 and 0.88 Pa.s.

At $15^{\circ} \mathrm{C}$ (Figure 2.b), the decrease in viscosity at low shear rate is of higher magnitude, from 390 to 263 Pa.s at $6 \cdot 10^{-3} \mathrm{~s}^{-1}$. The viscosity at high shear rate barely increases from 0.71 to 0.78 Pa.s. At 
$20^{\circ} \mathrm{C}$ (Figure 2.c), the viscosity at $6 \cdot 10^{-3} \mathrm{~s}^{-1}$ decreases from 220 to 82.5 Pa.s, whereas the viscosity at $200 \mathrm{~s}^{-1}$ significantly increases from 0.62 to $1.4 \mathrm{~Pa}$.s. It can first be concluded that $15^{\circ} \mathrm{C}$ is an optimal processing temperature providing a lower viscosity than at $10^{\circ} \mathrm{C}$, but still stable at high shear rate, which is not the case at $20^{\circ} \mathrm{C}$.

The viscosity changes in opposite direction at low and high shear rate promote the previous interpretation that different interactions predominate depending on the shear rate. If hydrodynamic interactions predominate at high shear rate, the results of Figure 2 show that the fluid phase viscosity increases with time as the result of a temperature activated mechanism occurring in the fluid phase, namely the polycondensation of the dissolved alumino-silicates with the silicates in solution. This mechanism requires that dissolution has started previously, which is consistent with the lower viscosity at low shear rate, governed by interactions between particles, as a result of a lower solid fraction of alumino-silicate particles.

\section{Rheological behavior of diluted suspensions}

Dilution of the non-aged suspension leads to a progressive decrease of the viscosity irrespective of the shear rate. The comparison of the diluted suspension rheograms exhibits a larger effect of dilution at high shear rate, which is consistent with the previous interpretation that viscosity of highly sheared suspensions is mainly governed by hydrodynamic interactions.

\section{Rheology evolution during ageing}

Ageing at $-18^{\circ} \mathrm{C}$ (Figure 4 ) results in a progressive and large decrease of the viscosity at low shear rate (from 390 Pa.s at $6 \cdot 10^{-3} \mathrm{~s}^{-1}$ to 1.48 Pa.s at $6 \cdot 10^{-2} \mathrm{~s}^{-1}$ ), together with a slight increase of the viscosity at $200 \mathrm{~s}^{-1}$, from 0.71 to 1.05 Pa.s, between ageing durations $\mathrm{T}_{0}$ and $\mathrm{T}_{5}$. This evolution follows the same trend as those of the non-aged suspension at $15^{\circ} \mathrm{C}$. However, polycondensation is not likely to occur at such a low temperature $\left(-18^{\circ} \mathrm{C}\right)$, and the increase in viscosity at high shear rate is more probably related to the increase in the amount of alumino-silicate in solution in the fluid phase along ageing. A long ageing time at $-18{ }^{\circ} \mathrm{C}$ results in a suspension rheology that tends toward a Newtonian behavior. If the viscosity at low shear rate is mainly governed by non-hydrodynamics interactions between particles, and if the decrease in viscosity at low shear rate is the result of the dissolution of alumino-silicate particles, it can be concluded that the silicate solution, in which alumino-silicate particles are dispersed, behaves as a Newtonian fluid. On the other hand, the non-Newtonian behavior is related to the presence of large alumino-silicate particles and vanishes once all aluminosilicate particles are dissolved. In the Discussion section, a phenomenological model is proposed, based upon these interpretations. These results show that ageing is an efficient way to adapt the 
suspension rheology to the requirements of liquid composite molding.

\section{Discussion}

\section{Phenomenological modeling}

Provided that the precursor under study is a suspension of alumino-silicate in a silicate solution ${ }^{1}$, it is assumed to be composed of a solid fraction $\phi_{1}$ of large alumino-silicate particles, dispersed in a solution of fine silicate particles of solid fraction $\phi_{2}$, with a fluid phase of viscosity $\eta_{f}$ as solvent. Assuming a full-scale separation between the scales of both particles populations and under such conditions that hydrodynamic effects predominate (i.e. at high shear rate), two Krieger-Dougherty models can be combined to express the suspension viscosity $\eta$, Eq. (1). In Eq. (1), $\eta_{f}\left(1-\frac{\phi_{2}}{\phi_{M}}\right)^{-[\eta] \phi_{M}}$ acts as the viscosity of the suspension of fine particles, in which large particles are dispersed ${ }^{16}$. $\eta=\eta_{f}\left(1-\frac{\phi_{2}}{\phi_{M}}\right)^{-[\eta] \phi_{M}}\left(1-\frac{\phi_{1}}{\phi_{M}}\right)^{-[\eta] \phi_{M}}$

It is further assumed that all particles are spherical, i.e. $[\eta]=2.5$, (no occurrence of high aspect ratio particles were evidenced by scanning electron microscopy observations of hardened samples of nonaged suspension), and that the maximal packing fraction, $\phi_{M}$, is the same for both suspensions of large and fine particles.

\section{Ageing}

The main asset of such a description is its suitability for describing the dissolution of the large particles, involved during ageing of the suspension, as a decrease in solid fraction of large particles, $\phi_{1}$, and an increase in solid fraction of fine particles, $\phi_{2}$, namely an amount of large particles is converted into fine particles. As no evidence of significant volume change during ageing was reported, dissolution is assumed to occur at constant global solid volume fraction. This leads to Eq. (2) for the relation between the solid fraction of fine particle, $\phi_{2}^{T}$, and the solid fraction of large particles, $\phi_{1}^{T}$, at ageing time $T$, as a function of the initial volume fractions.

$\phi_{2}^{T}=\phi_{2}^{T_{0}}+\frac{\phi_{1}^{T_{0}}-\phi_{1}^{T}}{1-\phi_{1}^{T}}\left(1-\phi_{2}^{T_{0}}\right)$

For long ageing times, it is assumed that all the large particles are dissolved, i.e. $\phi_{1}^{T \rightarrow \infty}=0$, and Eq. (2) can be written as Eq. (3).

$\phi_{2}^{T \rightarrow \infty}=\phi_{2}^{T_{0}}+\phi_{1}^{T_{0}}\left(1-\phi_{2}^{T_{0}}\right)$

\section{Dilution of the precursor}

The initial volume fractions are themselves function of dilution, Eq. (4) and (5), where $x=V_{w} / V$ is the volume dilution rate, i.e. the volume of added water, $V_{w}$, per volume of initial suspension, $V . \phi_{1}^{x=0}$ 
and $\phi_{2}^{x=0}$ are respectively the initial solid fraction of large particles in suspension and fine particles in solution, in the non-diluted precursor.

$\phi_{1}^{T_{0}}=\frac{\phi_{1}^{x=0}}{1+x}$

$\phi_{2}^{T_{0}}=\phi_{2}^{x}=0 \frac{1-\phi_{1}^{x=0}}{1-\phi_{1}^{x=0}+x}$

Dilution also affects the viscosity of the fluid phase, $\eta_{f}$, which is not just water. Actually, the suspension has undergone a short ageing period before dilution, and some large particles are thus dissolved, changing the nature of the fluid phase. In the diluted suspensions, the fluid phase is thus a mixture of the initial fluid phase (viscosity $\eta_{f}$ ) and of water (viscosity $\eta_{w}$ ). Many models, more or less complex, provide the viscosity of a mixture of liquids ${ }^{17-19}$. In the present case, the fluid phase is essentially water and it is not expected a significant difference between $\eta_{f}$ and $\eta_{w}$. An affine relation is thus simply assumed, Eq. (6), between $\eta_{f}$ and the dilution rate of the fluid phase, $x^{\prime}=V_{w} /\left(V_{f}+V_{w}\right)$ , where $V_{f}$ is the initial volume of fluid phase. $x^{\prime}$ is related to $x$ through Eq. (7).

$\eta_{f}=\left(1-x^{\prime}\right) \eta_{f}^{x^{\prime}=0}+x^{\prime} \eta_{w}$

$x^{\prime}=\frac{x}{x+\left(1-\phi_{1}\right)\left(1-\phi_{2}\right)}$

It is noteworthy that Eq. (6) usually involves logarithm of viscosities ${ }^{19}$. However more reliable results were obtained with Eq. (6) in the present case. Indeed using logarithms would require very low values (40\%) of the maximum solid fraction or very high intrinsic viscosity (8.8) for the fine particles. Investigating a more physically meaning model for mixture viscosity is still necessary but it is thought not to be of prior importance and it will not be carried out in this paper.

According to the Eyring theory ${ }^{18,19}$, the temperature dependence of the fluid phases viscosity is given in Eq. (8) and (9), respectively for water and initial fluid phase (with $E_{w}=1.90 \cdot 10^{4} \mathrm{~J} \cdot \mathrm{mol}^{-1}$ and $\eta_{w}^{0}$ $=4.02 \cdot 10^{-7}$ Pa.s). Previously, the non-hydrodynamic interactions between particles have been shown to be temperature dependent in the temperature range investigated, between 10 and $20^{\circ} \mathrm{C}$. Only the high shear rate viscosity, mainly governed by hydrodynamic interactions, will be further analyzed as a function of temperature.

$\eta_{f}^{x^{\prime}}=0=\eta_{f}^{0} e^{\frac{E_{f}}{R T}}$

$\eta_{w}=\eta_{w}^{0} e^{\frac{E_{w}}{R T}}$

\section{Calibration from rheology results}

Eq. (1) is unable to account for non-Newtonian behavior, as it requires that hydrodynamic interactions predominate. Those latter are likely to actually predominate for high shear rates, i.e. in the low viscosity range of the rheograms. The value of this low viscosity, $\eta_{\infty}$, is obtained for each 
rheogram by fitting the viscosity with a Carreau-Yasuda law, and Eq. (1) is now written as Eq. (10). In the high shear rate range, it is usually assumed that the packing fraction is $\phi_{M}=\frac{\pi}{3 \sqrt{2}}$.

$\eta_{\infty}^{T}=\eta_{f}\left(1-\frac{\phi_{2}^{T}}{\phi_{M}}\right)^{-[\eta] \phi_{M}}\left(1-\frac{\phi_{1}^{T}}{\phi_{M}}\right)^{-[\eta] \phi_{M}}$

Using the $\eta_{\infty}$ values for the non-aged reference suspension at 10,15 and $20^{\circ} \mathrm{C}$, together with values for diluted suspension at $15{ }^{\circ} \mathrm{C}$, makes it possible to assess the values of the unknown parameters, i.e. $\eta_{f}^{0}, E_{f}$ and $\phi_{2}^{T_{0}}$. Indeed, $\phi_{1}^{T_{0}}$ is directly deduced from $\phi_{2}^{T_{0}}$ using Eq. (3), provided that $\phi_{2}^{T \rightarrow \infty}$ is known. $\phi_{2}^{T \rightarrow \infty}$ is related to the viscosity at high shear rate of the suspension with high ageing time, $\eta_{\infty}^{T \rightarrow \infty}$, using Eq. (10) with $\phi_{1}^{T \rightarrow \infty}=0$, rewritten as Eq. (11).

$\eta_{\infty}^{T \rightarrow \infty}=\eta_{f}\left(1-\frac{\phi_{2}^{T \rightarrow \infty}}{\phi_{M}}\right)^{-[\eta] \phi_{M}}$

A Nelder-Mead algorithm is used to identify the values of $\eta_{f}^{0}, E_{f}$ and $\phi_{2}^{T_{0}}$. The viscosity values at high shear rate provided by the model are in good agreement with experiment, as shown in Figure 5. The values of the parameters are reported in Table 1. It is noteworthy that a similar agreement can be achieved with other assumptions (different values of intrinsic viscosity $[\eta]$ or maximal packing fraction $\phi_{M}$, using Quemada model instead of Krieger-Dougherty model), and those assumptions remains to be studied more in depth. However it is not the scope of the paper to account in detail of each phenomenon, but instead to build a global rheological framework.

\section{Thixotropic behavior}

Eq. (10) satisfactorily describes the high shear rate viscosity, which is mainly governed by hydrodynamic interactions. The description of the non-Newtonian behavior displayed in Figure 1-4 requires the introduction of a new feature to take into account other interactions at low shear rates. In a lack of any accurate knowledge on the interactions involved, a phenomenological approach is used. Several approaches allow implementing thixotropy in a rheological law such as Eq. (1). A microstructure variable, governed by a law of evolution, may be explicitly implemented in the model $^{11,20}$. The maximum packing fraction may be defined as varying as a function of the dominating interactions and consequently as a function of shear rate ${ }^{8,21}$. A third approach may interpret microstructure changes as build-up or breakdown of particles aggregates that can enclose a fluid volume that now acts as solid fraction ${ }^{22}$.

In the present work, an effective solid volume fraction, governed by an evolution law, is thus introduced in Eq. (1) instead of the actual solid volume fraction. In the present case, the nonNewtonian behavior vanishes after long ageing time. Moreover, ageing is mainly related to the 
dissolution of large particles, and it is therefore quite reasonable to use an effective solid fraction of large particles, $\phi_{e f f}$, to act as microstructure variable, Eq. (12). The silicate solution in which alumino-silicate particles are dissolved is assumed to be Newtonian, which is consistent with previous interpretations. A similar approach was proposed to take into account structural units in cement-based pastes ${ }^{22}$.

$\eta=\eta_{f}\left(1-\frac{\phi_{2}^{T}}{\phi_{M}}\right)^{-[\eta] \phi_{M}}\left(1-\frac{\phi_{e f f}}{\phi_{M}}\right)^{-[\eta] \phi_{M}}$

Thanks to Eq. (12), $\phi_{\text {eff }}$ can be assessed from suspension viscosity value provided that the other parameters are known, Eq. (13).

$\phi_{e f f}=\phi_{M}\left(1-\frac{\left(\frac{\eta}{\eta_{f}}\right)^{\frac{1}{-[\eta] \phi_{M}}}}{1-\frac{\phi_{2}^{T}}{\phi_{M}}}\right)$

A non-dimensional microstructure parameter is introduced, $s$, which describes the relative variation of $\phi_{\text {eff }}$, Eq. (14). A simple evolution law is assumed for $s$ as a function of strain rate, Eq. (15), which includes a build-up, $t_{b u}$, and breakdown, $t_{b d}$, characteristic time for microstructure change mechanisms.

$$
\begin{aligned}
& s=\frac{\phi_{e f f}-\phi_{e f f}^{\infty}}{\phi_{e f f}^{0}-\phi_{e f f}^{\infty}}=\frac{\eta_{\infty}^{\frac{1}{-[\eta] \phi_{M}}}-\eta^{\frac{1}{-[\eta] \phi_{M}}}}{\eta_{\infty} \frac{1}{-[\eta] \phi_{M}}-\eta_{0} \frac{1}{-[\eta] \phi_{M}}} \\
& \dot{S}=\frac{\dot{\phi}_{e f f}}{\phi_{e f f}^{0}-\phi_{e f f}^{\infty}}=-\frac{s}{t_{b d}}+\frac{1-s}{t_{b u}}
\end{aligned}
$$

The equilibrium microstructure parameter, $s_{e q}$, is achieved for a given shear rate if $\dot{s}=0$, leading to Eq. (16).

$\frac{t_{b u}}{t_{b d}}=\frac{1-s_{e q}}{s_{e q}}$

The equilibrium microstructure parameter is assessed using Eq. (14), with equilibrium viscosity values, $\eta$, as a function of shear rate. Here, $\eta$ is the average value of the steady viscosities measured at increasing and decreasing shear rate. A stretched exponential function, Eq. (17), is chosen to fit the experimental values and is in very good agreement with experiment for the reference suspension (Figure 6).

$s_{e q}=s_{e q}^{\infty}+\left(s_{e q}^{0}-s_{e q}^{\infty}\right) \cdot e^{-\left(\tau_{s} \dot{\gamma}\right)^{\beta}}$

The microstructure build-up characteristic time, $t_{b u}$, is assumed to be constant. The microstructure breakdown characteristic time, $t_{b d}$, function of the shear rate, is thus deduced from Eq. (16) and (17) 
if $t_{b u}$ is known. The value of $t_{b u}$ is identified from simulations of the rheological characterization cycle, in order to fit the steady viscosity values (change in stress lower than $5 \%$ during $5 \mathrm{~s}$ ) at increasing and decreasing shear rate. A microstructure build-up characteristic time, $t_{b u}=10 \mathrm{~s}$, allows a very proper agreement with the experimental steady viscosity at increasing and decreasing shear rate (Figure 7).

The ability of the thixotropic model to describe the transient viscosity of the reference suspension was checked on the basis of a test at a constant shear rate of $70 \mathrm{~s}^{-1}$. The initial value of the microstructure parameter was calculated to match the first viscosity value, and Eq. (15) was integrated to compute the time evolution of the microstructure parameter at constant shear rate, from which the transient viscosity is deduced. The kinetics computed for transient viscosity is very similar to the experimental viscosity, despite a discrepancy of about $5 \%$ between the viscosities at the end of the test (Figure 7).

\section{Conclusions}

The thixotropic rheological behavior of a geopolymer precursor suspension was investigated in the range of processing temperature $\left(10-20^{\circ} \mathrm{C}\right)$. At high shear rate, hydrodynamic effects seems to predominate and the effective viscosity is directly related to the fluid phase viscosity. At low shear rate, the effective viscosity is of 2 or 3 orders of magnitude higher because of the influence of other interactions, as Brownian effects. Ageing at $-18^{\circ} \mathrm{C}$ inhibits the polycondensation mechanism but dissolution of alumino-silicate particles may occur. It results in a large decrease of effective viscosity at low shear rate, whereas the viscosity at high shear rate is almost unchanged. As a consequence, the rheology of the suspension tends toward a Newtonian behavior at long ageing time.

On the basis of these interpretations of rheology changes and geopolymerization mechanism, a phenomenological model is proposed. It includes two solid fractions (large particles suspended in a solution of fine particles) and combines two Krieger-Dougherty equations to describe the effective viscosity of the suspension, assuming a full scale separation. Calibration of the model is performed based on the viscosity at high shear rate of reference and diluted suspensions. Dissolution of large particles is described as a conversion of an amount of large particles into the same volume of fine particles.

A scalar microstructure parameter is introduced as an effective solid fraction of large particles. It is governed by a simple evolution law, which involves two build-up and breakdown mechanisms. Once identified using equilibrium viscosity data, the model is able to reproduce the hysteresis between increasing and decreasing shear rate viscosity, as well as transient viscosity at constant shear rate. Many features of the model are based on assumptions that are still to validate, but the overall model 
provides a framework suitable to describe the rheological changes of the precursor while ageing. A more accurate knowledge of the formulation would provide a deeper insight on the mechanisms at work, and therefore a more accurate description of the different phenomena.

\section{Acknowledgment}

Part of this work was carried out in the framework of the collaborative project COMPTINN' funded by OSEO-DGCIS within the field of the ASTech and Aerospace valley competitiveness clusters.

\section{References}

[1] Davidovits J. Geopolymer, Chemistry and Applications. Saint-Quentin, France: Institut Géopolymère; 2008. ISBN: 9782951482098

[2] Xu H, Van Deventer JSJ. The geopolymerisation of alumino-silicate minerals. Int J Miner Process. 2000;59:247-266. https://doi.org/10.1016/S0301-7516(99)00074-5

[3] Palomo A, Banfill R, Fernandez-Jimenez A et al. Properties of alkali-activated fly ashes determined from rheological measurements. Adv Cem Res. 2005;17:143-151.

https://doi.org/10.1680/adcr.2005.17.4.143.

[4] Bourlon A. Physico-chimie et rhéologie de géopolymères frais pour la cimentation des puits pétroliers. PhD thesis, Université Pierre et Marie Curie, France; 2010. (in french)

[5] Nazockdast E, Morris JF. Active microrheology of colloidal suspensions: simulation and microstructural theory. Journal of Rheology. 2016;60:733. https://doi.org/10.1122/1.4954201. [6] Pednekar S, Chun J, Morris J. Bidisperse and polydisperse suspension rheology at large solid fraction. Journal of Rheology. 2017;62:513. https://doi.org/10.1122/1.5011353.

[7] Deboeuf S, Lenoir N, Hautemayou D et al. Imaging non-Brownian particle suspensions with X-ray tomography: application to the microstructure of Newtonian and visco-plastic suspensions. Journal of Rheology. 2018;62:643. https://doi.org/10.1122/1.4994081.

[8] Jamali S, Boromand A, Wagner $\mathrm{N}$ et al. Microstructure and rheology of soft to rigid shearthickening colloidal suspensions. Journal of Rheology. 2015;59:1377. https://doi.org/10.1122/1.4931655

[9] Sielbert LE, Melrose JR. The rheology and microstructure of concentrated, aggregated colloids. Journal of Rheology. 1999;43:673. https://doi.org/10.1122/1.551028.

[10] Barnes HA. Thixotropy - a review. J Non-Newton Fluid. 1997;70:1-33. https://doi.org/10.1016/S0377-0257(97)00004-9.

[11] Baravian C, Quemada D, Parker A. Modelling thixotropy using a novel structural kinetics approach: basis and application to a solution of iota carrageenan. J Texture Stud. 1996;27:371-90. 
https://doi.org/10.1111/j.1745-4603.1996.tb00082.x.

[12] Mujumdar A, Beris AN, Metzner AB. Transient phenomena in thixotropic systems. J Non-Newton Fluid. 2002;102:157-78. https://doi.org/10.1016/S0377-0257(01)00176-8.

[13] de Souza Mendes PR. Modeling the thixotropic behavior of structured fluids. J Non-Newton Fluid. 2009;164:66-75. https://doi.org/10.1016/j.jnnfm.2009.08.005.

[14] Castillo HA, Wilson HJ. Elastic instabilities in pressure-driven channel flow of thixotropicviscoelasto-plastic fluids. J Non-Newton Fluid. 2018;261:10-24.

https://doi.org/10.1016/j.jnnfm.2018.07.009.

[15] Farrugia A. Relations procédé-microstructure-comportement de composites à matrice vitrocéramique mis en oeuvre par voie liquide. PhD thesis, University of Toulouse, France; 2013. (in french)

[16] Faris RJ. Prediction of the viscosity of multimodal suspensions from unimodal viscosity data. Trans Soc Rheol. 1968;12:281-301. https://doi.org/10.1122/1.549109.

[17] Viswanath DS, Ghosh TK, Prasad DHL, Dutt NVK, Rani KY. Viscosity of Liquids. Theory, Estimation, Experiment, and Data. Dordrecht, Germany: Springer; 2007.

https://doi.org/10.1007/978-1-4020-5482-2

ISBN 978-1-4020-5481-5

[18] Bloomfield VA, Dewan RK. Viscosity of liquid mixtures. J Phys Chem. 1971;75:3113-3119. https://doi.org/10.1021/j100689a014.

[19] Weirong JI, Lempe DA. Calculation of viscosities of liquid mixtures using Eyring's theory in combination with cubic equations of state. Chinese J Chem Eng. 2006;14:770-779. https://doi.org/10.1016/S1004-9541(07)60010-X

[20] Tiu C, Boger D. Complete rheological characterization of time-dependent food products. J Texture Stud. 1974;5:329-338. https://doi.org/10.1111/j.1745-4603.1974.tb01439.x.

[21] van der Sman RGM, Vollebregt HM. Effective temperature for sheared suspensions: A route towards closures for migration in bidisperse suspension. Adv Colloid Interface Sci. 2012;185-186:113. https://doi.org/10.1016/j.cis.2012.08.006.

[22] Toutou Z, Lanos C, Mélinge Y et al. Modèle de viscosité multi-échelle: de la pate de ciment au micro-béton. Rhéologie. 2004;5:1-9. 


\section{Figure captions}

Figure 1. Effect of temperature on the rheological behavior of non-aged suspension at increasing (solid lines) and decreasing (dashed lines) shear rate. The dotted lines plot the low shear rate and low torque limits of the rheometer.

Figure 2. Evolution of the rheological behavior in the range of processing temperature, at (a) 10, (b) 15 and (c) $20^{\circ} \mathrm{C}$, and at increasing (solid lines) and decreasing (dashed lines) shear rate. 25 minutes have past between the start of two consecutive cycles. The dotted lines plot the low shear rate and low torque limits of the rheometer.

Figure 3. Effect of a 2, 3 or $5 \mathrm{wt} . \%$ water dilution on the rheological behavior of the suspension at $15^{\circ} \mathrm{C}$ and at increasing (solid lines) and decreasing (dashed lines) shear rate. The dotted lines plot the low shear rate and low torque limits of the rheometer.

Figure 4. Effect of ageing at $-18^{\circ} \mathrm{C}$ on the rheological behavior of the suspension at $15^{\circ} \mathrm{C}$ and at increasing (solid lines) and decreasing (dashed lines) shear rate. The dotted lines plot the low shear rate and low torque limits of the rheometer.

Figure 5. Comparison of high shear rate viscosity, $\eta_{\infty}^{T}$, obtained from Carreau-Yasuda law fits of the rheograms (o symbols), and from Eq. (3-10) with parameter values in Table 1 (surface).

Figure 6. Equilibrium microstructure parameter of the reference suspension as a function of shear rate obtained from experiment (o symbols) via Eq. (13), and fit with a stretched exponential function, Eq. (17) (line).

Figure 7. Comparison of modeled viscosities (lines) with experiment (o symbols): (left) steady viscosity values at increasing and decreasing shear rate, and (right) transient viscosity at shear rate of $70 \mathrm{~s}^{-1}$.

\section{Tables}

Table 1. Identified values of the unknown parameters.

\begin{tabular}{lrrr}
\hline$\eta_{f}^{0}$ (Pa.s) & $E_{f}\left(\mathrm{~J} \cdot \mathrm{mol}^{-1}\right)$ & \multicolumn{1}{c}{$\phi_{2}^{T_{0}}(-)$} & $\phi_{1}^{T_{0}}(-)$ \\
\hline $1.56 \cdot 10^{-7}$ & $2.87 \cdot 10^{4}$ & 0.189 & 0.568
\end{tabular}




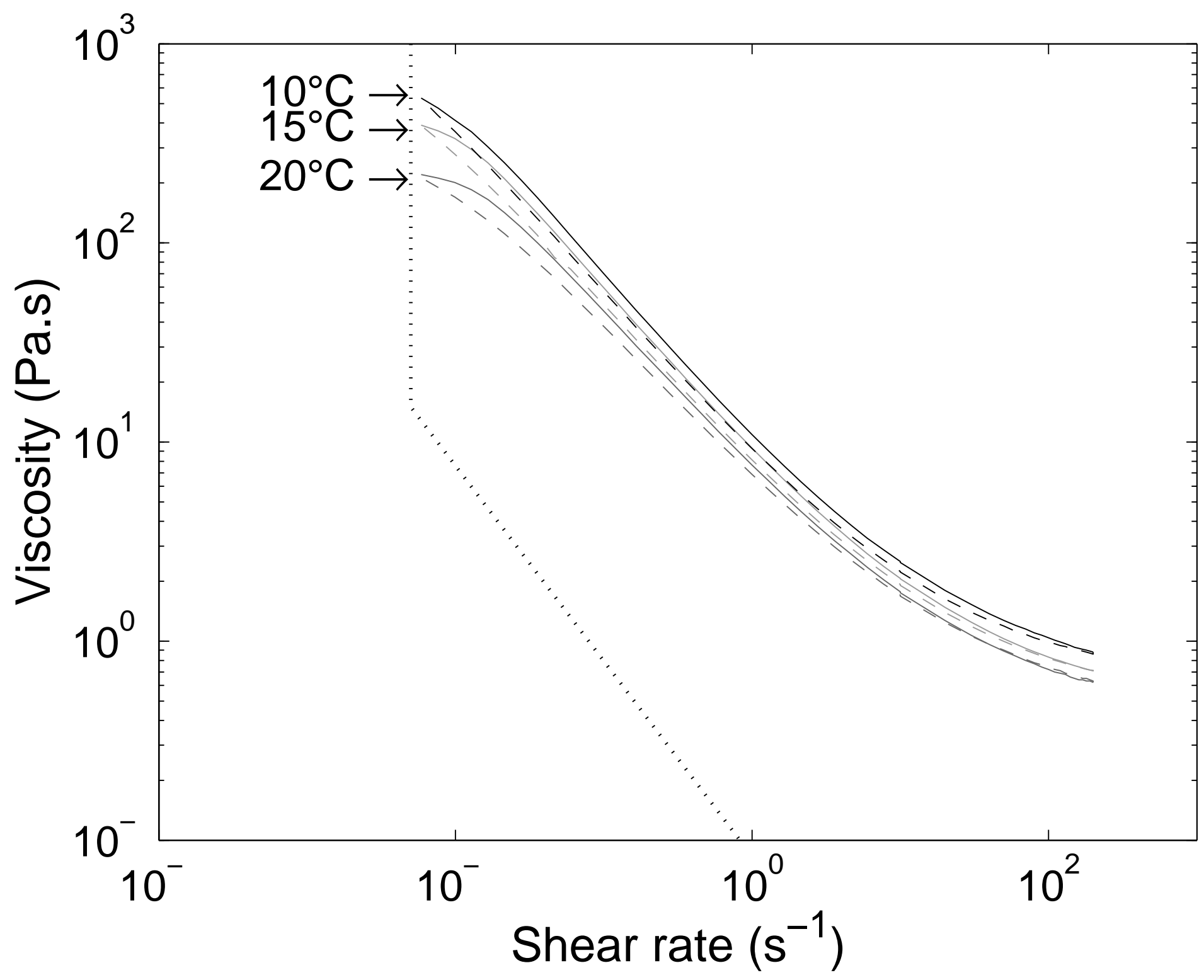

ijac_13508_f1.eps 


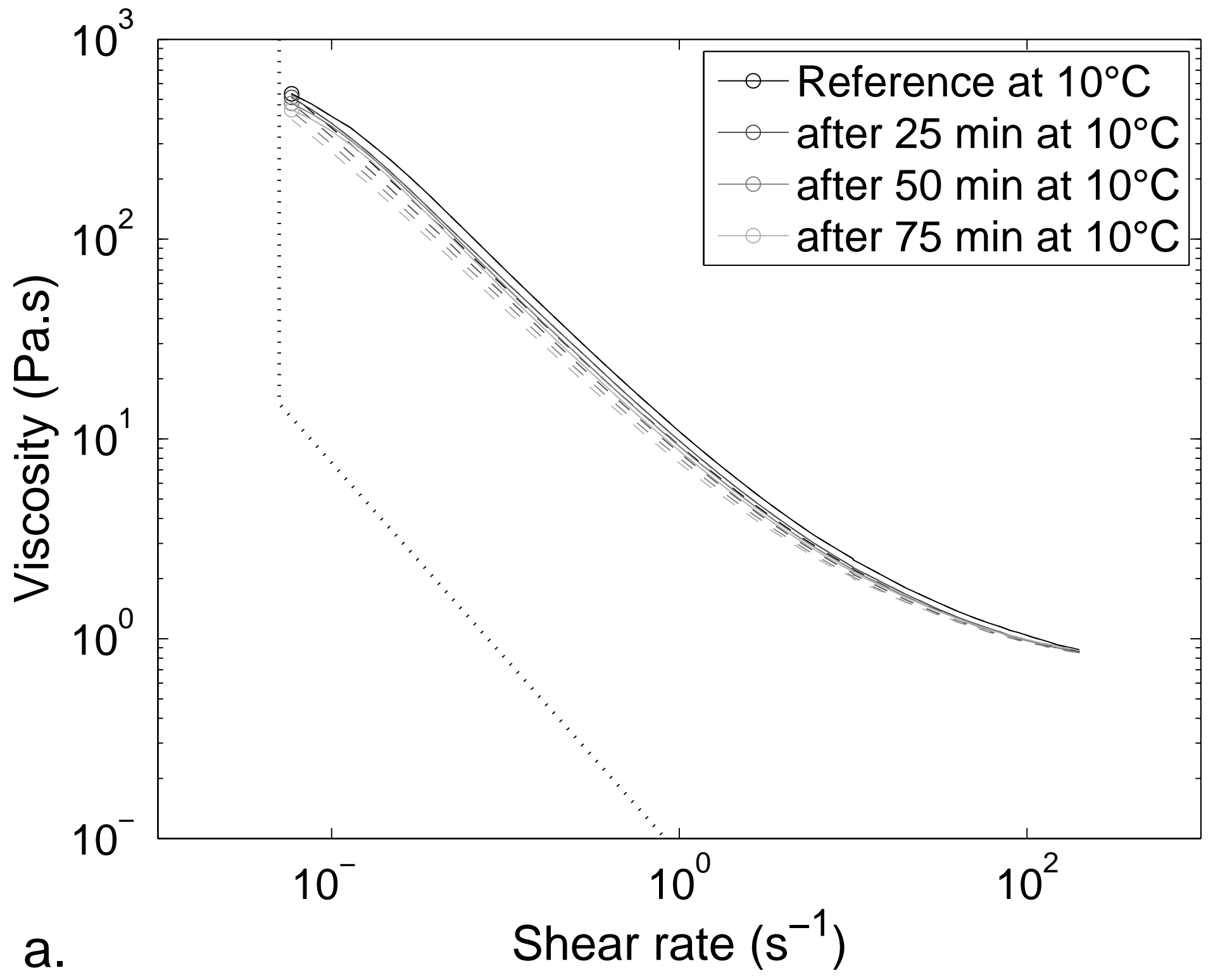

ijac_13508_f2a.eps 


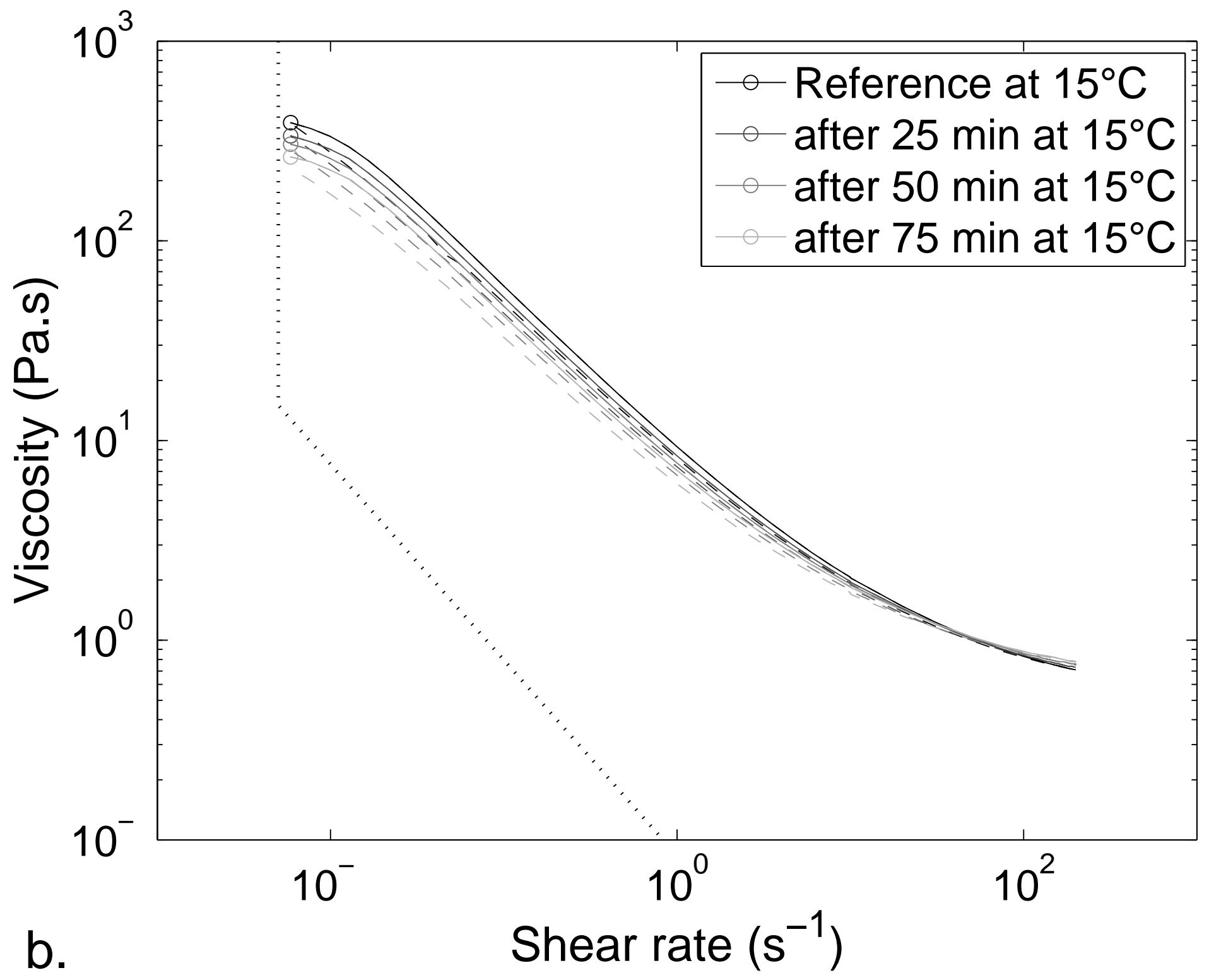

ijac_13508_f2b.eps 


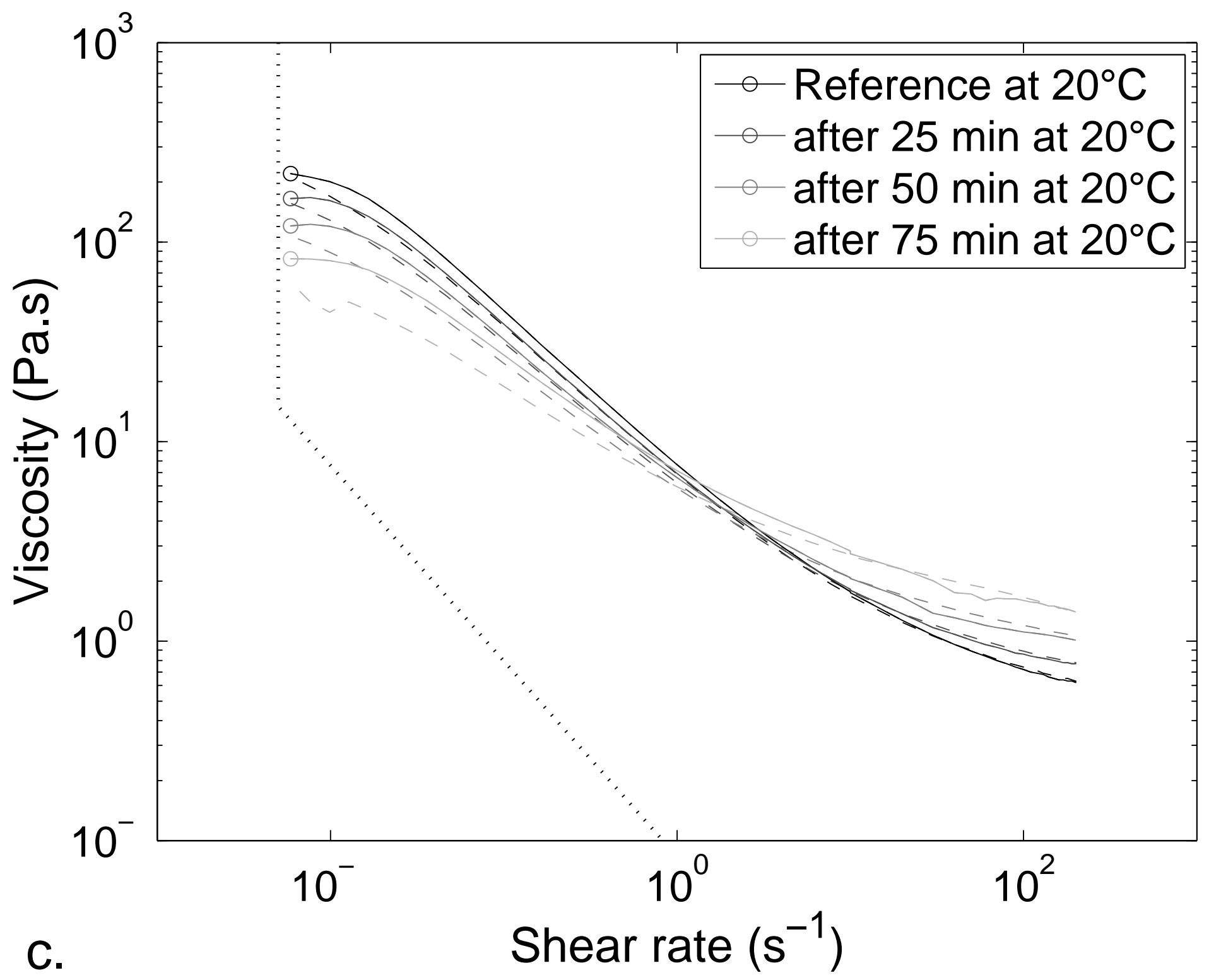

ijac_13508_f2c.eps 


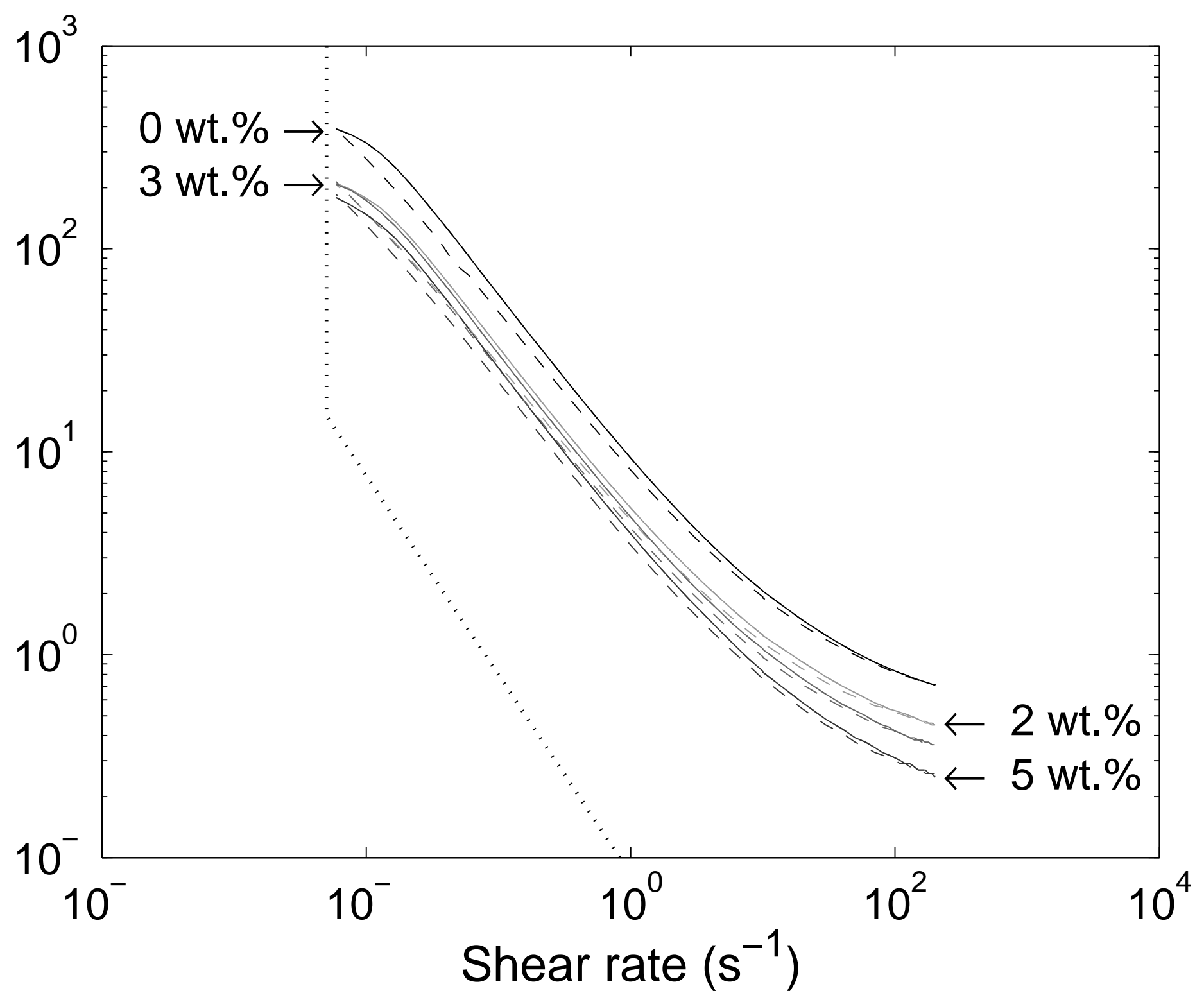

ijac_13508_f3.eps 


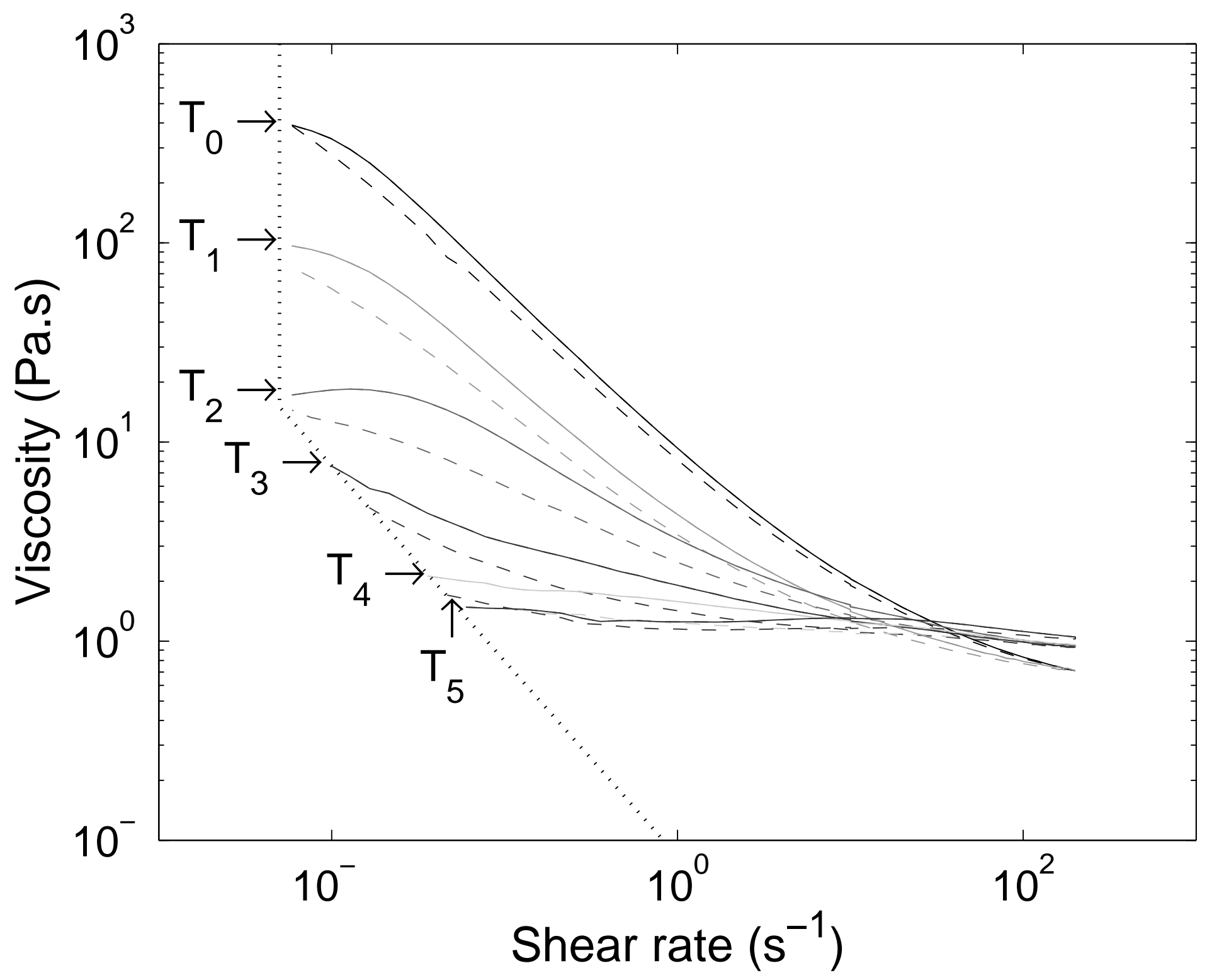

ijac_13508_f4.eps 


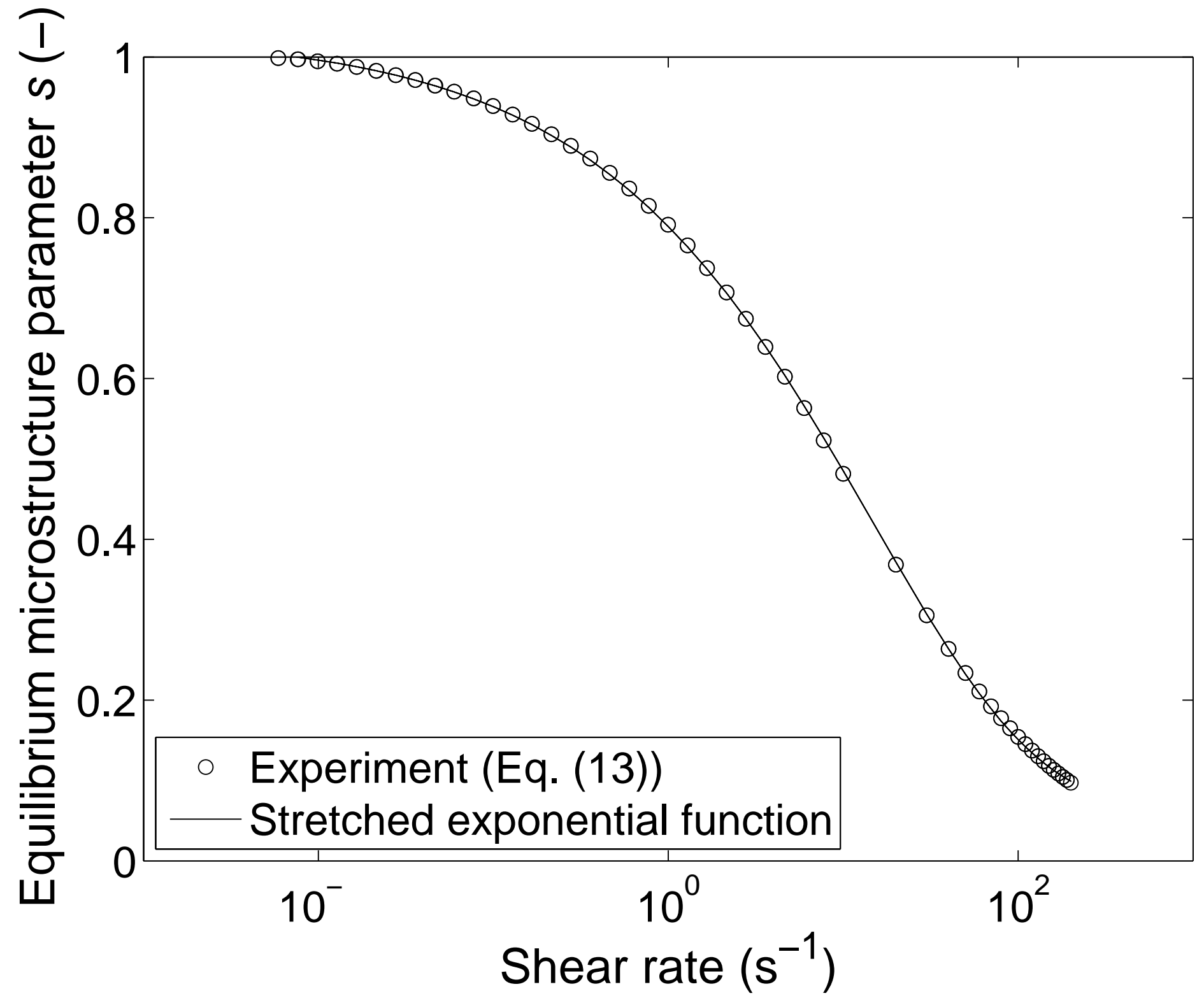

ijac_13508_f6.eps 


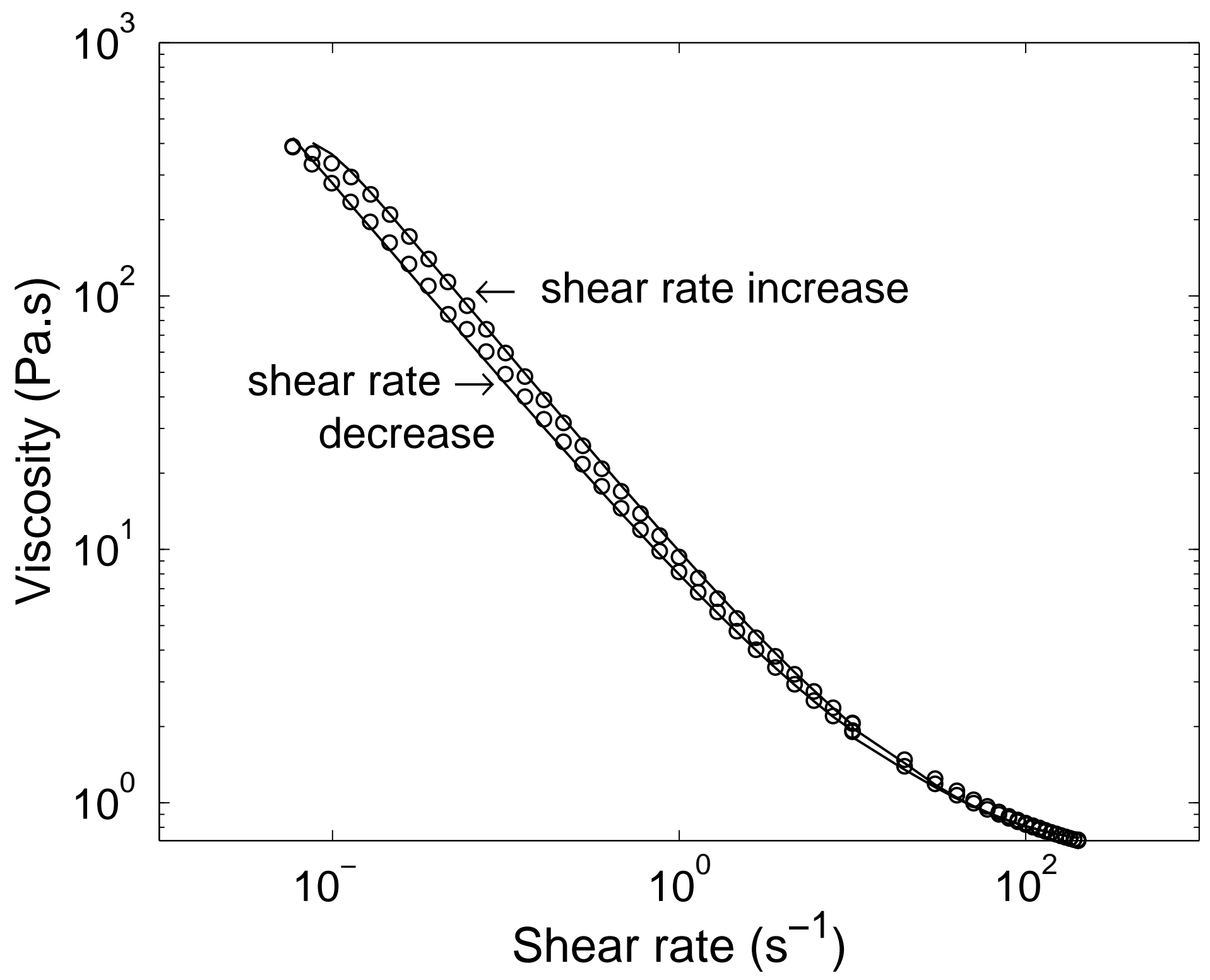

ijac_13508_f7a.eps 


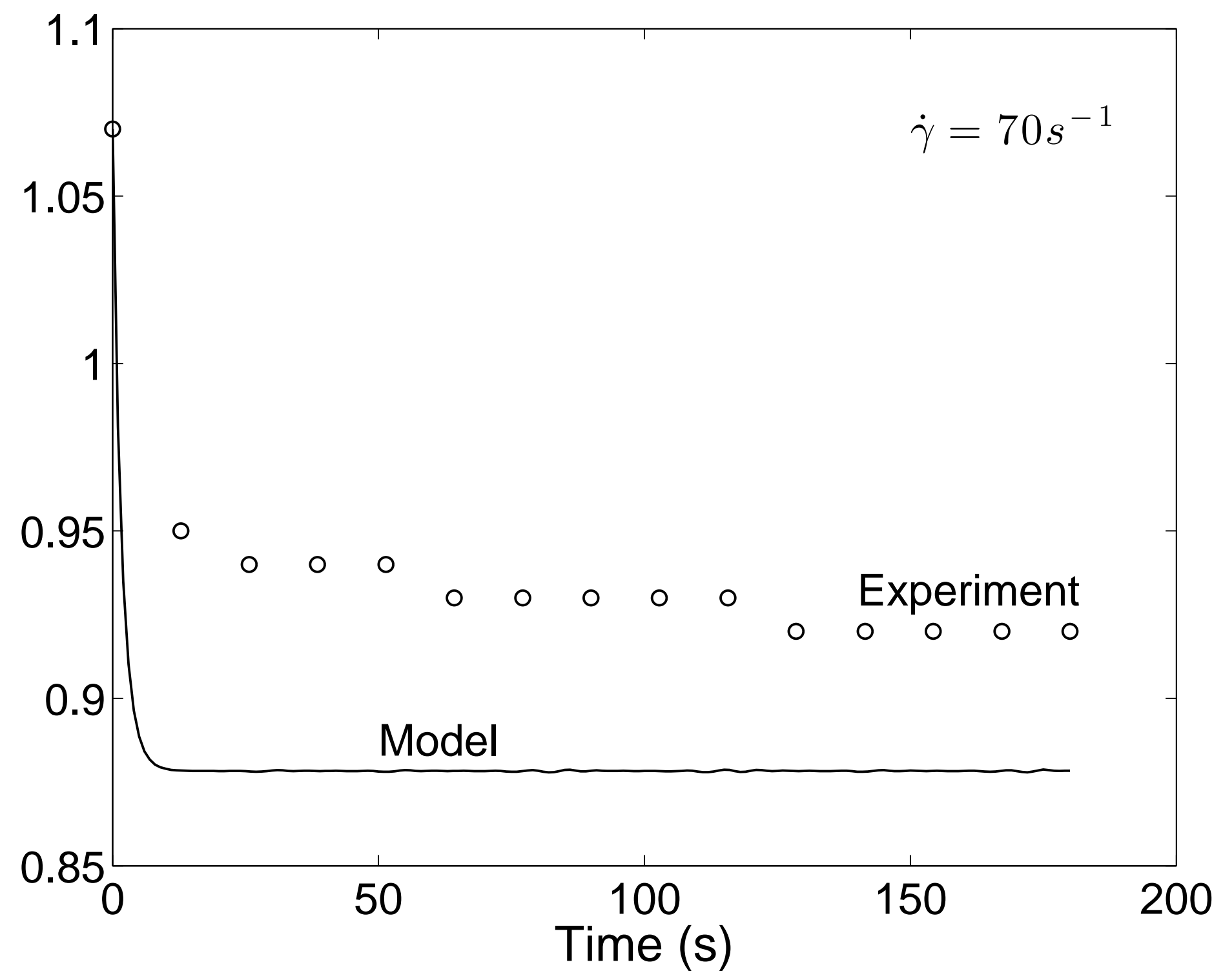

ijac_13508_f7b.eps 